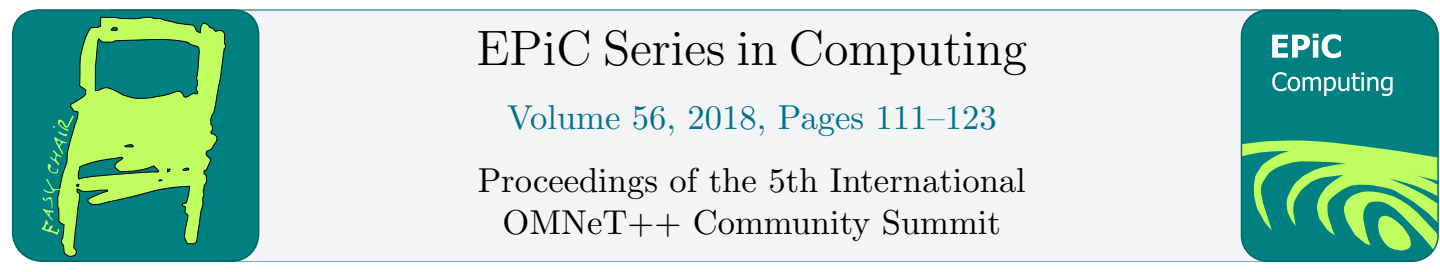

\title{
Teaching Modelling and Analysis of Communication Networks using OMNeT++ Simulator
}

\author{
Koojana Kuladinithi, Raphael Elsner, Leo Krüger, Sebastian Lindner, \\ Christoph Petersen, Daniel Plöger, Zeynep Vatandas, Andreas Timm-Giel \\ Institute of Communication Networks, Hamburg University of Technology, Germany \\ koojana.kuladinithi@tuhh.de
}

\begin{abstract}
At the Hamburg University of Technology, Germany, the modelling of communication networks using the OMNeT++ simulator and the INET framework is taught for Master students. Teaching the concepts of simulation and modelling while letting the students obtain a hands-on experience during a 14 -week (4h/week) period (single semester) is a challenging task. The diversity of the pre-knowledge of the participating students and the duration of the course are the main challenges that need to be addressed when organising such a course. This paper discusses the structure of this course and the best practices followed. The course adopts a methodology where lectures on concepts are mixed with INET based exercises that begin with simple topics and gradually moving into advanced topics.
\end{abstract}

\section{Introduction}

Easy access to information, exponential growth of information stored in the Internet and rapid technological progress require to rethink our teaching concepts and methods practiced at Universities for centuries. Students have to be trained more on methods of scientific and engineering work than on learning information by heart, which will have changed over the next decade. Therefore, Hamburg University of Technology has undergone a dramatic change of teaching towards more problem and project based learning with larger teaching modules. In this context, the Simulation and Modelling of Communication Networks course offered for computer science and electrical engineering master programs has been redesigned as a project based learning module.

Simulation is a common method for engineers and computer scientists in their profession. Unfortunately, the theoretical background is not always well understood when using simulation tools and the results are not often critically questioned and evaluated. In this course, our main objective therefore is to teach the theoretical background of simulations in general and simulation and modelling of communication networks more specifically. We decided to use only one simulation tool as an example and have students actively work with it. So they can apply and reflect on theoretical backgrounds in aspects such as random number generators, pp. $111-123$ 
mobility, radio channel or traffic models, directly with the simulation tool. Further, they can analyse and discuss their own results. Besides the subject-oriented expertise, engineers and computer scientists need to be able to work on specific tasks in teams. Therefore, we organise the exam in a way that a team of two students work on a specific task and present their results together. More specifically, we follow the German implementation of the European Qualification Framework [1] to define the teaching objectives. In short, this course is organised to achieve the following four outcomes:

1. Theoretical Knowledge: Students obtain all the basic concepts of discrete event simulation and network performance evaluation.

2. Capabilities: Students are able to get used to network simulation for performance evaluation of communication networks. The students are able to analyse the obtained results and explain the effects observed in the network. Further, they are able to question their own results.

3. Social Competence: Students are able to acquire expert knowledge in groups, present the results, discuss solutions and different approaches. They are able to work out solutions for new problems in small teams.

4. Autonomy: Students are able to work self-reliantly. They are able to tackle problems they come across by themselves, and show that they continuously question results.

In order to achieve the above outcomes, many challenges have to be addressed: The most challenging part of the whole course is how to cater our lecture contents and exercises to suit students with heterogeneous backgrounds. Since this course is offered for both German as well as International Master's programs, the enrolled students usually come from different countries with different academic disciplines. In general, most of the students have neither used a simulator nor have learned about simulation concepts such as discrete event simulation, random number generation, etc. Further, they usually have completed their Bachelors degrees at different universities and hence, not all of them have the basic knowledge required for this course. The main prerequisites of this course are the knowledge of computer and communication networks, and basic programming skills.

This course was introduced to the program syllabus in 2010. We have gone through several iterations of our lecture contents and OMNeT ++ and INET based exercises during the last five years to improve based on experiences gained and feedback from both students and tutors. To the best of our knowledge, we as tutors, believe that the structure of this course is at a good standard to achieve the above four mentioned outcomes. Therefore, we focus in this paper on explaining our lecture structure, focusing more on the exercises based on OMNeT ++ simulator and INET framework [2]. We believe that this paper provides the OMNeT++ community insights in to how best to organise such a course. The use of OMNeT++ simulator for teaching purpose was cited only once in 1999 [5], to the best of our knowledge.

The contents of this paper are organised as follows. Section 2 shows how we structure this course to fulfil the course requirements during the 14 -week (4h/week) period. The challenges we face are also being discussed. The next section gives a very detailed explanation to our exercises based on the OMNeT++ simulator and the INET framework. Section 4 details how we organise the final examination and the last section concludes the paper. 


\section{Structure and Content of the Course}

Our students are mainly graduate students who have followed either computer science or electrical engineering disciplines. Therefore, the lecture contents are organised assuming that all the students have a basic knowledge of computer and communication networks. For example, the details of OSI model and protocols like TCP are not part of the course.

Every week of this course consists of three parts - the first part with 60 to 90 minutes duration is allocated for teaching, the second part with 60 minutes is allocated for group discussions focussing on the approach and the results of the previous exercise and in the third part with 90 minutes duration, a new exercise is introduced. Table 1 shows our curriculum for 2018. The exercises are done in smaller groups, each consisting of six to eight students.

The optimum number of students that one tutor can supervise is usually six to eight based on our experiences. After the lecture, the students work in their own group, each group is supervised by one tutor in a separate room equipped with all required facilities for discussions including a beamer, white board, etc. Two students work together as a team to complete the exercise and contributing for the discussion held after each exercise.

Table 1: Simulation and Modelling of Communication Networks Curriculum - 2018

\begin{tabular}{|l|c|l|}
\hline \multicolumn{1}{|c|}{$\begin{array}{c}\text { Part 1 } \\
\text { 90 minutes }\end{array}$} & \multicolumn{1}{|c|}{$\begin{array}{c}\text { Part 2 } \\
\text { 60 minutes }\end{array}$} & \multicolumn{1}{c|}{$\begin{array}{c}\text { Part 3 } \\
\text { 90 minutes }\end{array}$} \\
\hline \hline $\begin{array}{l}\text { Lecture 1: Simulation Ba- } \\
\text { sics }\end{array}$ & OMNeT++ Introduction and installation \\
\hline Lecture 2: Stochastics & \multicolumn{1}{|c|}{ Exercise 1: Tic-Toc Tutorial } \\
\hline $\begin{array}{l}\text { Lecture 3: Random Num- } \\
\text { ber Generation }\end{array}$ & \multicolumn{1}{|c|}{ Exercise 2: Probability Distributions } \\
\hline $\begin{array}{l}\text { Lecture 4: Analysis of Re- } \\
\text { sults }\end{array}$ & Discussion on exercise 2 & $\begin{array}{l}\text { Exercise 3: Random Num- } \\
\text { ber Generator }\end{array}$ \\
\hline $\begin{array}{l}\text { Lecture 5: Simulation } \\
\text { Models }\end{array}$ & Discussion on exercise 3 & $\begin{array}{l}\text { Exercise 4: INET Frame- } \\
\text { work Introduction }\end{array}$ \\
\hline $\begin{array}{l}\text { Discussion on Results } \\
\text { Analysis }\end{array}$ & Discussion on exercise 4 & $\begin{array}{l}\text { Exercise 5: Results Anal- } \\
\text { ysis }\end{array}$ \\
\hline $\begin{array}{l}\text { Lecture 6: Hypothesis } \\
\text { Testing }\end{array}$ & Discussion on exercise 5 & $\begin{array}{l}\text { Exercise 6: Distribution } \\
\text { Fitting }\end{array}$ \\
\hline \multicolumn{2}{|c|}{ Exercise 7: Wide Area Network Task } \\
\hline $\begin{array}{l}\text { Lecture 7: Wireless Net- } \\
\text { works }\end{array}$ & Exercise 7: Wide Area Network Task \\
\hline \multicolumn{2}{|c|}{ Presentations and group discussions on exercise 7 } \\
\hline $\begin{array}{l}\text { Lecture 8: Advanced Top- } \\
\text { ics in Simulations }\end{array}$ & Exercise 8: Wireless Local Area Networks \\
\hline Discussion on exercise 8 & \multicolumn{1}{|c|}{ Distribution of the final task } \\
\hline
\end{tabular}

The details of the exercises based on the OMNeT++ simulator and the INET framework are given in Section 3. Two main references of the lecture contents are [3] and [4]. Here are the contents of our lecture in detail. 
- Lecture 1 - Simulation Basics: Details the discrete event simulation approaches (classifications of systems and simulations, simulation via Discrete Events, Future Event List, etc).

- Lecture 2 - Stochastics: Overview of the elementary of probability theory (random variables, different probability distributions, expectations, means and variances, etc).

- Lecture 3 - Random Number Generation: Details the basics of random number generation (methods used to generate random numbers, base generator, different random number generators, generating discrete and continuous random variables, etc).

- Lecture 4 - Statistical Analysis of Simulation Results: Details the analysis of result evaluation (sample mean, sample variance, interval estimates of a population mean).

- Lecture 5 - Simulation Models: Details three models used in a simulator: traffic models, mobility models and radio propagation models

- Lecture 6 - Hypothesis Testing: Details the $\chi^{2}$ test to prove that the results follows the expected distribution. Examples are given to show the equi-probable and equi-distance approaches used in the $\chi^{2}$ test.

- Lecture 7 - Wireless Networks: Details the behaviour of Carrier Sense Multiple Access/ Collision Avoidance (CSMA/CA) used in IEEE 802.11 based networks.

- Lecture 8 - Advanced Topics in Simulations: Details how to speed up the simulation using parallel simulations and statistical variance reduction techniques like the use of common random numbers. This lecture also discusses how to select and validate the simulation parameters before running simulations in bigger scenarios.

The workload of this course is estimated as 70 hours of study time during the semester spread over 14 weeks and 110 hours of independent study time. This course is worth six ECTS (European Credit Transfer and Accumulation System) credit points for the students. The final examination is done as an oral examination. More details about the final examination are given in Section 4. The main challenges we faced when organising this course are:

Challenge 1 - High resource utilisation: This course needs more physical and human resources. At least 4 rooms are required to support all enrolled students during the exercises. Further, all the tutors should have experience in using the OMNeT++ simulator and the INET framework. Therefore, we also have a special guide for each exercise for new tutors to refer to.

Challenge 2 - Heterogeneous backgrounds of students: As the students come from different disciplines and different universities, some do not have the required knowledge to understand the lecture contents. As a solution, we provide a script for each lecture consisting of recommended references that a student must refer for more details. We have also introduced the lecture 7 - Wireless Networks to discuss CSMA/CA in detail as we noticed that most of the students are not capable of analysing the behaviour of wireless networks.

Challenge 3 - Lack of programming knowledge: If a student does not have sufficient knowledge of $\mathrm{C}++$ programming, we as tutors offer to help, but we do not teach $\mathrm{C}++$ in this course.

Challenge 4 - Students' work habits: This course is not designed for students who plan to pass the exam without attending the lectures and doing the exercises. It is extremely hard to complete our final task designed for the examination, if a student does not know about the simulator. At the beginning of this course, we clearly explain to the students that they should attend the course regularly. This creates an additional challenge on us to keep up the motivation of the students. We also consider student attendance and the contribution to the discussion 


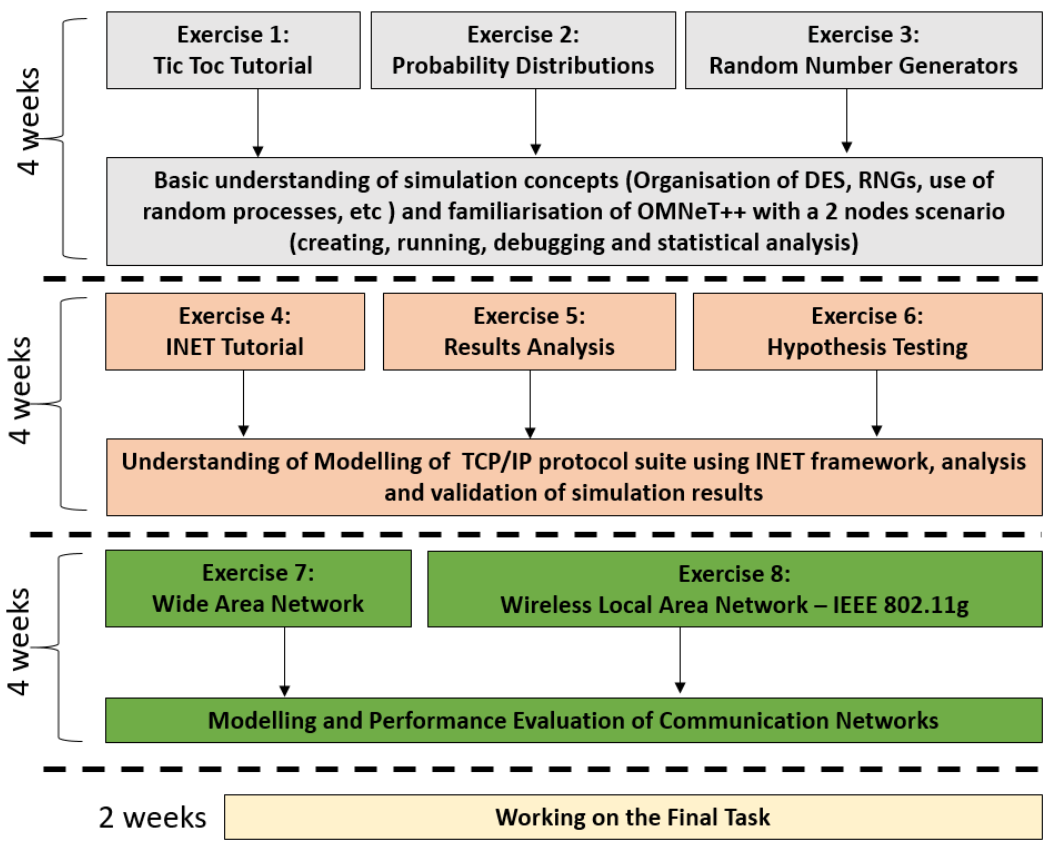

Figure 1: Organisation of Exercises

during the exercises for their final grading. We, as tutors, are also motivated to contribute to this course to have very capable students when they start their research and thesis projects later. The students who attended this course can easily work with the OMNeT++ simulator and also feel comfortable to learn how to use another network simulator quickly.

\section{Organisation of Exercises}

Our exercises are structured to cover three main areas: first, the students should understand the basic concepts of a simulator, second, understand how the TCP/IP protocol suite is modelled in a simulator and third, how to analyse a TCP/IP based communication network. As shown in Figure 1, all eight exercises are covered within the first 12 weeks and the last two weeks are allocated to start with the final task. After the completion of exercises, the students are expected to have a sound knowledge of using the OMNeT++ simulator with the INET framework to analyse a communication network. Our experiences have shown that the 12week period of 150 minutes per week, assisted by tutors, is insufficient to complete the work. Therefore, we expect the students to allocate extra one to two hours of time every week for this course. The complete description of all the exercises are available at [6].

\subsection{Understanding Simulation Concepts}

During the first week of our course, the students are given an introduction to the OMNeT++ simulator and are asked to install the simulator on their own laptops to start with the exercise 1. The Tic-Toc tutorial in OMNeT++ [2] is used as our first exercise in order to understand the simulator. 
Learning Targets of exercise 1: Setting up an OMNeT++ project, Adding the network and omnetpp.ini files, Launching and running the project, Debugging and statistics collection.

In exercise 2, the students are asked to create a simple model consisting of a sender $S$ and a receiver $R$. The sender creates new messages with a random time interval $T$ and sends it to the receiver. The receiver records the inter-arrival time of the messages.

Learning Targets of exercise 2: Understand the lecture 2 - Stochastics by analysing the PDF and CDF of the inter-arrival times of different distributions, How to refer to the $\mathrm{OMNeT}++$ manual and understand the parameters used in models, Compare the results w.r.t. a lower and higher number of samples or simulation durations, Compare the mean and variance of simulation results with theoretical computations.

Exercise 3 is an extension to exercise 2, focussing on random number generators (RNG). Exemplarily, the students should implement the Linear Congruential random number Generator $(L C G)$ and use it to generate the aforementioned inter-arrival time $\mathrm{T}$ (as in exercise 2). The students are asked to compare the results with the ones using the OMNeT++ default RNG (Mersenne Twister) and in addition, they are encouraged to discuss on the characteristics of "good" random number generators.

Learning Targets of exercise 3: Deeper understanding of the theory learned in lecture 3 - RNGs by implementing an own RNG (e.g., LCG generator), Investigate the effect on results of different RNGs (seed, period), Getting used to modifying $\mathrm{C}++$ code.

After completing the first three exercises, the students should have a good foundation to understand the basic concepts of a simulator and to get used to another simulator faster.

\subsection{Use of INET Framework}

Exercise 4 shows how to start with the INET framework by building a simple network. The StandardHost module is used as a client and a server and PPP is used as a link layer to configure the channel parameters such as transmission rate, delay and BER.

Learning Targets of exercise 4: Becoming familiar with TCP/IP based models,Understand the difference between vector and scalar files, Analyse the TCP throughput observing how TCP congestion control algorithm works, See the impact on the upper layer performance by changing the link parameters.

The objective of exercise 5 is to understand the importance of the warmup period and confidence intervals for the evaluation of simulation results. Exercise $\mathbf{5}$ uses the same network as in Exercise 4, but with different settings. 


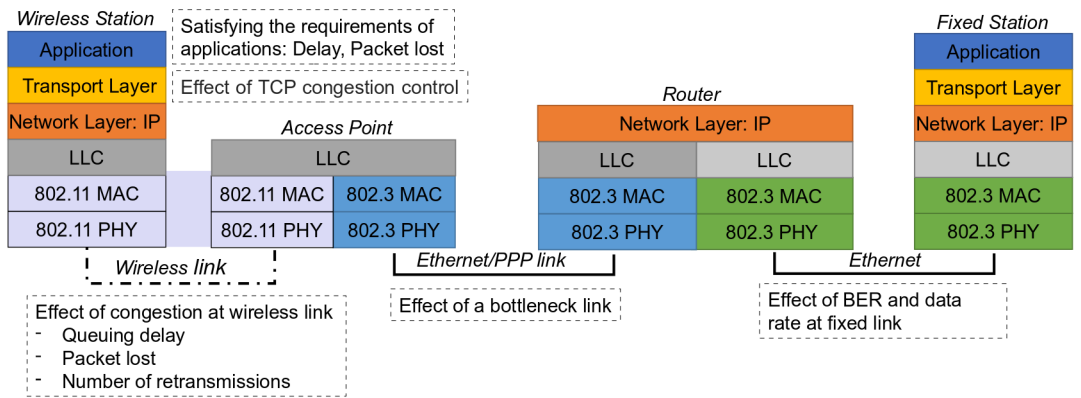

Figure 2: The protocol stack, requirements and the effects considered when evaluating communication networks

Learning Targets of exercise 5: Deeper understanding of lecture 3 - Analysis of Results and lecture 5 - Simulation Models, Visualise the effect of the warmup period due to the TCP slow start phase, Understand the difference between vector and scalar files, Observe the variation of the size of the confidence interval when increasing the number of runs and number of samples used in a single run.

The focus of exercise 6 is to cater to more complex types of traffic in a simulator as taught in lecture 6. For example, a constant data transmission should not be expected as used in exercise 5, but a user who retrieves web contents and needs some time for reading before making the next request. These time periods also need to be configured in the simulation model, so that the user behaviour is represented properly. Exercise 6 explains the method of distribution fitting, which can be used to create a probability distribution based on empirical data. The goodness of fit is evaluated by using the $\chi^{2}$ (chi-squared) test. The students are asked first to describe the $\chi^{2}$ test in pseudo-code and implement it in MATLAB. Then the $\chi^{2}$ test is used to validate the simulation results obtained for the Poisson distribution on the incoming packet rates.

Learning Targets of exercise 6: Know how to use empirical data in a simulator, Deeper understanding of the goodness of fit test learned at lecture 6 - Hypothesis Testing, Use of MATLAB functions.

\subsection{Modelling and Evaluation of Communication Networks}

After completing the first six exercises, the students should have a good understanding of using $\mathrm{OMNeT}++$ and the INET framework to simulate a simple scenario, and to perform a detailed analysis. We also encourage students to use additional tools like MATLAB to analyse the results in this course. The last two exercises are focused on investigating how a simulator should be used to evaluate a communication network. As shown in Figure 2, we focus on evaluating a network by investigating how the change of parameters at the transport layer and link layer (both wireless and wired) affects the performance of the applications. Therefore, exercise 7 is focused on analysing a complex wide area network and exercise $\mathbf{8}$ is focused on teaching how an IEEE 802.11 based wireless network is modelled. 


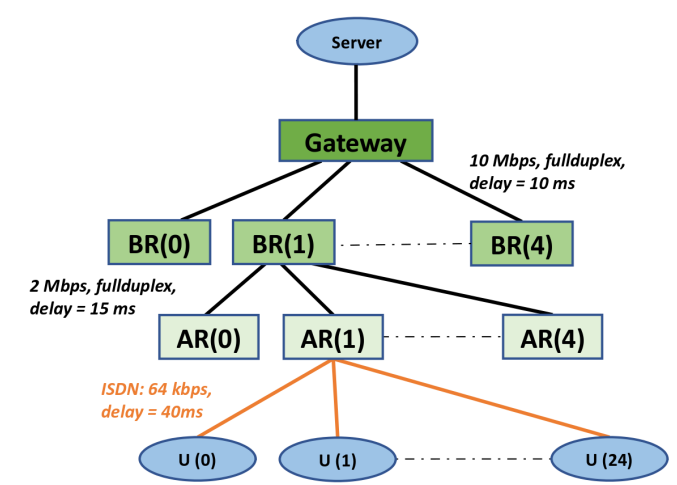

Figure 3: A network topology of the exercise 7: Wide Area Network

Before starting exercise 7, we conduct a session called the Hidden Beauty of OMNeT++ to highlight basic and some advanced features of the simulator such as "Use of CMDenv, How to choose a random seed, the difference between Vector files and Scalar files, How to show the plots in OMNeT++, How to put the results into different directories, How to define different configurations in one ini file and how to run parallel simulations using our servers to make students feel comfortable in using the $\mathrm{OMNeT}++$ simulator.

The structure of exercise $\mathbf{7}$ is different compared to the previous exercises. The students are given a 2-week period to complete this task and the third week is allocated to presenting their results to the group. Exercise $\mathbf{7}$ is very helpful for students to prepare for the final examination. The following paragraph shows a part of the description.

"A network operator contacted your team and asked for consultancy. Currently the operator is running a small Internet Protocol (IP) network with simple data services using Integrated Services Digital Network (ISDN) with 64 kbps. Besides the data services he would like to offer a simple Voice over IP (VoIP) service to selected customers. The simple voice service should enable customers to make phone calls to other customers within the operator's network. Further, the operator thinks about upgrading all ISDN dial up nodes to DSL (Digital Subscriber Line). You as a communication engineer, is asked to support the operator's decision process by giving him the performance figures he requires"

As shown in Figure 3, exercise 7 consists of 4 backbone routers and 24 access routers each serving maximum of 25 VoIP users. The students are asked to propose a reasonable number of active VoIP users that an access router can support guaranteeing a 1\% of packet loss rate including the dropping of packets which exceeds a delay of $200 \mathrm{~ms}$. The task is to support the operator's decision process by giving him the performance figures required. In this exercise, the students usually come up with different worst case scenario analysis by varying number of VoIP users selecting different pairs of communications in the given topology. The group discussions held during the third week of this task are very dynamic as different teams have different ways of looking at the problem and coming up with different proposals on how to improve the quality of VoIP application. 
Learning Targets of exercise 7: Understand a text description and model it in a simulation, Justify the simulation parameters used (e.g., simulation duration, warmup period, number of repetitions, etc), Add new statistics such as packet lost due to higher delay, Modify application protocols, Use the simulation models to analyse the worst case scenario, Give a presentation justifying the results.

The last exercise is focused on understanding the behaviour of a wireless network. The students are asked to create one stationary wireless host connected to the WLAN access point and communicating with another host on the Internet. In this exercise, the connection between the access point and the host on the Internet is replaced by a switch and a router letting students to understand how routing works.

Learning Targets of exercise 8: Understand the behaviour $C S M A / C A$ using a simple network, Configuration of required parameters used in WLAN, Analysis of results by investigating IEEE 802.11 based statistics, Setting up the routing table.

\section{Final Examination}

The final task is given to the students at the 13th week of their study time. The task is designed for students to put in one to two weeks of full-time effort. Two students work together in a team to complete the final task, which is similar to exercise $\mathbf{7}$, but organised to analyse a complete different networking scenario involved with three different types of applications. The part of the $\mathbf{2 0 1 8}$ final task description is given below.

Final Task Description - 2018: A laboratory on autonomous vehicle technology wants to evaluate autonomous truck clustering. A truck cluster consists of a single cluster head $(\mathrm{CH})$ and several cluster member trucks. As shown in Figure 4, a special truck in front acts as $\mathrm{CH}$. The $\mathrm{CH}$ controls the platoon behaviour and gives control instructions through wireless communication. For this it has a wireless access point (WAP) mounted on its roof. To prevent packet loss the WAP packet queue is rather generously sized and can hold up to 50 packets.

The WAP is connected through a $100 \mathrm{Mbit} / \mathrm{s}$ cable connection to the $\mathrm{CH}$ router, which in turn is connected via a Long Term Evolution (LTE) radio link to the test lab router at the test laboratory. We can simplify this link as a point-to-point radio link with no loss, $10 \mathrm{~ms}$ packet delay and a data rate of $8 \mathrm{Mbit} / \mathrm{s}$.

Cluster members request control information from the $\mathrm{CH}$ through Hypertext Transfer Protocol (HTTP) requests via 802.11g wireless local area networking (WLAN). The HTTP requests have a fixed size of 200 Byte and are sent periodically every $100 \mathrm{~ms}$. The requested control information consist of Global Positioning System (GPS) position, speed, acceleration and planned movement actions (steering). The CH sends corresponding HTTP replies with a fixed size of $400 \mathrm{kB}$, containing the requested control information. The HTTP server is connected to the cluster head through a $100 \mathrm{Mbit} / \mathrm{s}$ Ethernet cable with the delay modelled as exponentially distributed with the mean of $30 \mathrm{~ms}$.

In front of the $\mathrm{CH}$ a special sensor car is driving, equipped with sensors to capture a 3D model of the environment. The raw 3D model data is continuously sent via the WAP and the point-to-point radio link to the test lab using User Datagram Protocol (UDP). The data is processed at the test lab. 


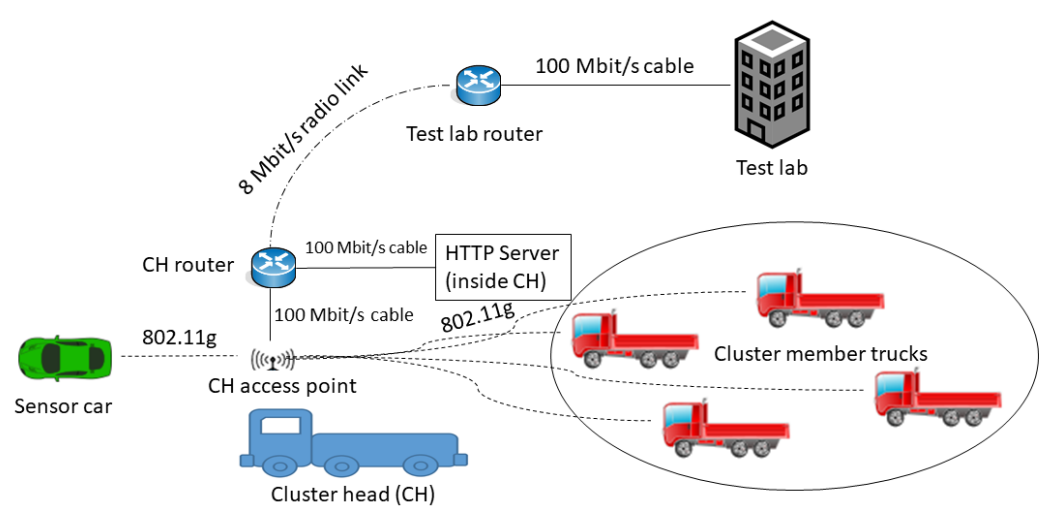

Figure 4: Final Task 2018 - Infrastructure view of the scenario

The UDP connection is bidirectional because cached environment data is also sent from the test lab to the sensor car so that the cluster can be made aware of upcoming road conditions.

This UDP stream comes with two strict requirements for both directions: first, packets should take no longer than $150 \mathrm{~ms}$; second, a packet loss of no more than $10 \%$ can be accepted. Note that packets discarded due to a delay larger than $150 \mathrm{~ms}$ also contribute to the total packet loss.

The 3D data packet sizes depend on the environment and are not constant. A trace file is provided to you that contains real-world measurements of these data packet sizes. Together with the trace file you will also be given the time interval between sending packets. The UDP stream server at the test lab is connected to the test lab router through a $100 \mathrm{Mbit} / \mathrm{s}$ Ethernet cable with the delay modelled as exponentially distributed with the mean of $30 \mathrm{~ms}$.

Additionally, one car inside the platoon is used to model a continuous transmission of a large file using File Transfer Protocol (FTP) between the cluster and the test lab. The FTP server is connected to the test lab router through a Ethernet cable with $100 \mathrm{Mbit} / \mathrm{s}$ and constant delay of $5 \mathrm{~ms}$.

\subsection{Tasks Involved}

The objective of the evaluation is to judge whether the infrastructure in place is sufficient for the given network traffic. Therefore, the students should first convert the described scenario into a simulation model, and gather data through simulations. The following questions should be addressed in the final report.

- How can 3D data packet sizes be modelled, based on the trace file?

- How many cluster members can be supported with the infrastructure so that the transmission of $3 \mathrm{D}$ sensor data is within the allowed range?

- How does the FTP transmission affect the behaviour?

- Is the LTE radio link dimensioned appropriately?

The two team members in a team should divide the task as follows:

1. One member should evaluate the scenario for the uplink case where the FTP connection uploads data from a truck to the test lab. 
2. The other member should evaluate the scenario for a downlink FTP connection from the test lab to the truck.

The outcome of each team's results varies as we use different parameters for modelling the 3D sensor data. Figure 5 shows one of the expected outcomes for the tasks explained above, when using the scenario with the FTP upload. We do not reveal all the details of the analysis here as our students might read this publication. However, to understand the reason for having lower packet losses at the sensor lab, with the increasing number of cluster members that results in higher HTTP load in the wireless network is not straight forward. This is a very challenging task to answer for students if they do not have the proper understanding of the protocol behaviour as shown in Figure 2.

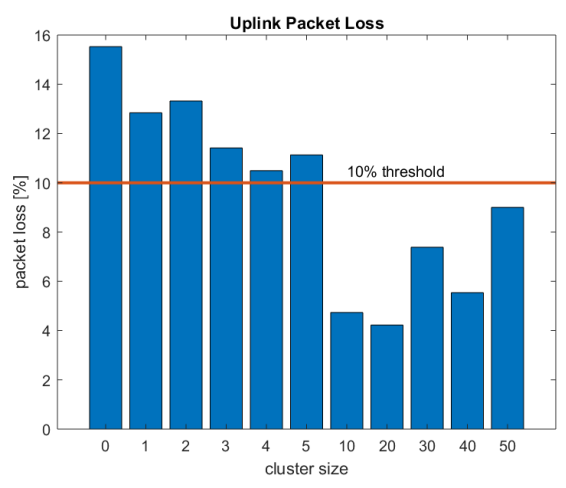

(a) At the sensor car

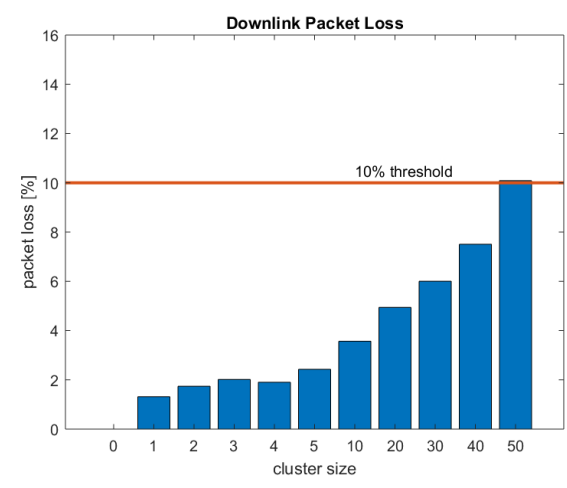

(b) At the test lab

Figure 5: Total packet loss rate of 3D sensor data

\subsection{Organisation of Examination}

After the end of the semester, the students are given another 4-week period before the oral examination to complete the work. We also provide consultation hours for students to meet tutors for any clarifications during this period. Before the examination date, the students are asked to provide a written report containing 10 to 20 pages and the slides for the presentation at the oral examination. We do not expect any implementation details and code in the report, but a detailed analysis of the results with justifications should be included in the report. Each team submits one report, indicating the separation of tasks between the team members.

We allocate each team one hour for the examination. Within this time, the students have a maximum of 30 minutes to jointly present their results. After the presentation, there are 20 minutes of questions for both students to ask further on their respective work done and also from the theory learned. The overall grade is decided based on the quality of the results, the presentation, the submitted report, the ability to answer questions in regard to the presentation and lecture contents and the participation in the exercises.

\section{Conclusion}

Teaching of the Simulation and Modelling of Communication Networks course is conducted for Master Students at the Hamburg University of Technology, Germany as a project 
Table 2: Students' Feedback given by 2016 Course Participants

\begin{tabular}{|l|c|c|c|}
\hline & Agree & Partly Agree & Disagree \\
\hline \hline $\begin{array}{l}\text { In this course, I learn something that I consider } \\
\text { to be important }\end{array}$ & $62 \%$ & $19 \%$ & $19 \%$ \\
\hline $\begin{array}{l}\text { The connection between individual teaching and } \\
\text { learning contents is in my view clearly apparent }\end{array}$ & $50 \%$ & $19 \%$ & $31 \%$ \\
\hline $\begin{array}{l}\text { Exercises that are presented in the course make } \\
\text { the theory content more comprehensible for me }\end{array}$ & $62 \%$ & $15 \%$ & $23 \%$ \\
\hline $\begin{array}{l}\text { During the course I have an opportunity to dis- } \\
\text { cuss teaching/learning content with others or to } \\
\text { ask questions }\end{array}$ & $73 \%$ & $23 \%$ & $4 \%$ \\
\hline $\begin{array}{l}\text { As a result of this course, I have learned how to } \\
\text { better make it clear to others what I mean }\end{array}$ & $45 \%$ & $25 \%$ & $30 \%$ \\
\hline $\begin{array}{l}\text { As a result of this course, I am now better able } \\
\text { to share and coordinate tasks with others }\end{array}$ & $52 \%$ & $29 \%$ & $19 \%$ \\
\hline $\begin{array}{l}\text { My ability to convert theoretical basics into prac- } \\
\text { tical applications is promoted by this course }\end{array}$ & $48 \%$ & $33 \%$ & $19 \%$ \\
\hline
\end{tabular}

based learning course. In a project based learning course, one of the requirements is to let students learn in an interactive manner instead of the traditional way of teaching where the students are pure listeners. A project based learning course let students work in smaller groups encouraging discussions, not only with the lecturer/tutor but also with peers. Furthermore, this results in enriching the quality of team work and gaining self confidence in presenting own ideas.

As highlighted in Section 2, this is a tough course to pass, if a student does not follow all the exercises. Our experiences have shown that some of the students find it difficult to get used to working with a simulator and also in applying what they have understood in the lecture, concepts like random number generations, hypothesis testing and the statistical analysis of the results. We usually encourage students first to discuss with team members and also with peer students in the group to find a solution to the problem on their own. Table 2 shows a part of students' feedback received in 2016.

All our exercises and the full description of the final task 2018 are available in a git repository [6]. We make sure all our exercises are running smoothly on the latest version of OMNeT++ and the INET framework available before the start of a new semester. The current versions we use are $O M N e T++$ 5.2.1 and INET 3.6.4. We are glad to support any research institute or University to share our lecture and exercise contents to conduct such a course.

\section{Acknowledgement}

We acknowledge our former colleagues, Jonas Eymann, Chunlei An and Lothar Kreft who have intensively contributed to enhance our lecture contents and the exercises of this course. 


\section{References}

[1] Information about courses, work-based learning and qualifications. Web page. Visited 2018-07-16 https://ec. europa.eu/ploteus/en/content/descriptors-page

[2] OMNeT++ Discrete Event Simulator. Web page. Visited 2018-06-21 https://omnetpp.org

[3] Averill M. Law and David Kelton, Simulation Modeling and Analysis, 4th ed. McGraw-Hill Higher Education, 1999.

[4] Sheldon M. Ross, Simulation, 4th ed. Academic Press, Inc., Orlando, FL, USA, 2006.

[5] Varga A., Using the OMNeT++ discrete event simulation system in education, in IEEE Transactions on Education, vol. 42, no. 4, pp. 11 pp, Nov. 1999. doi: 10.1109/13.804564

[6] Kuladinithi, K., Elsner, R., Krüger, L., Lindner, S., Petersen, C., Plöger, D., Vatandas, Z. and Timm-Giel, A. (2018, August). Teaching Modelling and Analysis of Communication Networks using OMNeT++ Simulator: Exercises and Final Task, Zenodo. http://doi.org/10.5281/zenodo. 1402067 